\title{
Doppler Tomographic Observations of Kepler-13b
}

\author{
Marshall C. Johnson and William D. Cochran \\ Department of Astronomy and McDonald Observatory, The University of Texas at Austin \\ 2515 Speedway, Stop C1400, Austin, TX 78712 USA \\ email: mjohnson@astro.as.utexas.edu, wdc@astro.as.utexas.edu
}

\begin{abstract}
We present Doppler tomographic observations of the transiting planet Kepler-13b (aka KOI-13b), a highly inflated hot Jupiter orbiting the $T_{\text {eff }}=8500 \mathrm{~K}$ primary of a hierarchical triple stellar system. As the planet transits the rapidly rotating host star, it successively blocks regions of the stellar disk with different radial velocities, causing a "bump" in the stellar spectral line shape, which we resolve spectroscopically. The manner in which this perturbation moves across the stellar line during the transit gives information on the relative alignment between the stellar spin and planetary orbital angular momentum vectors. This is a powerful statistical probe of planetary migration processes, as the expected spin-orbit misalignment distributions from dynamically cold migration (disk interactions) and dynamically hot migration (planetplanet scattering, Kozai cycles) are significantly different: the former will lead to primarily aligned orbits; the latter to a wide distribution. Doppler tomography also promises to be a powerful technique for confirming transiting planet candidates around rapidly rotating stars like Kepler-13A, which are not amenable to radial velocity follow-up and thus are currently a poorly sampled region of parameter space.
\end{abstract}

Keywords. (stars:) planetary systems, stars: individual: Kepler-13, techniques: spectroscopic

\section{Introduction}

Winn et al. (2010) found that hot Jupiters orbiting stars with $T_{\text {eff }}>6250 \mathrm{~K}$ tend to have orbital angular momentum vectors misaligned with respect to the stellar spin axis, while those orbiting stars with $T_{\text {eff }}<6250 \mathrm{~K}$ tend to be aligned. Several mechanisms have been proposed to explain this dichotomy. Winn et al. (2010) proposed a model of dynamically hot migration followed by tidal damping around cool stars. Batygin (2012) argued for a model of migration within a misaligned disk created by interactions with transitory binary companions in the birth cluster, followed by tidal damping. Rogers et al. (2012) instead proposed that transport of angular momentum by gravity waves within massive stars can cause changes in the rotation of the stellar atmosphere, resulting in an apparent misalignment of an initially aligned population. Observations of a larger sample of transiting planets across a wider range of parameter space will help to determine which of these scenarios is correct.

Kepler-13 (aka KOI-13, BD+46 2629) is a visual binary consisting of two similar Atype stars separated by $\sim 1^{\prime \prime}$ (Szabó et al. 2011). A transiting planet candidate orbiting the brighter star was identified by Kepler, and was confirmed through the detection of beaming and ellipsoidal variations by Shporer et al. (2011), who measured the mass of the planet to be $9.2 \pm 1.1 M_{J}$. The spin-orbit misalignment of the planet was measured using the gravity-darkened lightcurve caused by the rapid rotation and dynamical oblateness of Kepler-13A to be $\lambda= \pm 24^{\circ} \pm 4^{\circ}$ or $\pm 156^{\circ} \pm 4^{\circ}$ (Barnes et al. 2011). The degeneracy between prograde and retrograde orbits results from the fact that the stellar disk intensity profile is symmetric across the rotation axis. 


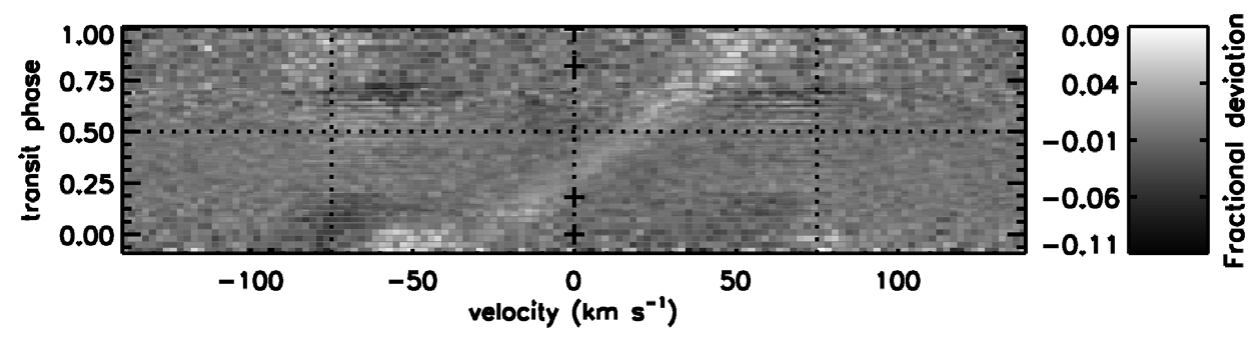

Figure 1. Time series line profile residuals combining all seven transits of Kepler-13b. The transit signature is the bright streak moving from bottom left to upper right. Time increases from bottom to top. Vertical dashed lines mark $v=0$ and $v= \pm v \sin i$, a horizontal dashed line marks the time of mid-transit, and small crosses mark the times of first through fourth contacts.

\section{Observations, Analysis, and Results}

Observations of Kepler-13b were obtained using the 9.2-m Hobby-Eberly Telescope (HET) and the High Resolution Spectrograph (HRS) at McDonald Observatory. We obtained 107 spectra during seven partial transits. Due to its fixed-altitude design the HET can only observe part of any given transit, but the lack of sensitivity of Doppler tomography to nightly radial velocity offsets allows us to stitch together multiple partial transit observations. The data were reduced using standard IRAF tasks.

We analyze the data using Doppler tomographic methods (e.g., Collier Cameron et al. 2010). Unlike traditional Rossiter-McLaughlin observations, where the distortions of the spectral lines during transit are interpreted as an anomalous velocity shift, we resolve the line profile perturbation spectroscopically. We use the least squares deconvolution method of Donati et al. (1997) to extract the time series line profiles. Transit parameters ( $v \sin i, \lambda, b, R_{p} / R_{*}$ ) are extracted by fitting a model produced by numerically integrating intrinsic line profiles across the visible disk of the star. One complication is the binary companion Kepler-13B; at a separation of $1^{\prime \prime}$, blended light from both stars enters the $2^{\prime \prime}$ HRS fibers, diluting the transit signal. The line profile of Kepler-13B also moves due to its orbital motion about its own stellar companion; our model includes a second line profile, moving according to the orbital parameters from Santerne et al. (2012).

The time series line profile residuals for Kepler-13b are shown in Fig. 1. We measure transit parameters of $\lambda=-21.3^{\circ} \pm 0.2^{\circ}$ and $b=0.772 \pm 0.002$. Note that the quoted formal errors are likely to be underestimates due to the low signal-to-noise of our detection and systematic errors. A Markov chain Monte Carlo code is under construction to address this. We confirm the Barnes et al. (2011) measurement of $\lambda$, and break the degeneracy in the gravity darkening results, showing that the orbit is prograde.

\section{References}

Barnes, J. W., Linscott, E., \& Shporer, A. 2011, ApJS, 197, 10

Batygin, K. 2012, Nature, 491, 418

Collier Cameron, A., et al. 2010, MNRAS, 407, 507

Donati, J.-F., Semel, M., Carter, B. D., Rees, D. E., \& Collier Cameron, A. 1997 MNRAS, 291, 658

Rogers, T. M., Lin, D. N.C., \& Lau, H. H.B. 2012, ApJ (Letters), 758, L6

Santerne, A., Moutou, C., Barros, S. C.C., et al. 2012, A\&BA, 544, L12

Shporer, A., Jenkins, J. M., Rowe, J. F., et al. 2011, AJ, 142, 195

Szabó, G. M., Szabó, R., Benkő, J. M., et al. 2011, ApJ (Letters), 736, L4

Winn, J. N., Fabrycky, D., Albrecht, S., \& Johnson, J. A. 2010, ApJ (Letters), 718, 145 